\title{
Introduction to the special issue
}

\author{
Manel Baucells • Antoni Bosch-Domènech • \\ Franz H. Heukamp
}

Published online: 15 December 2009

(C) Springer Science+Business Media, LLC. 2009

We were pleased to organize the FUR XIII conference. As organizers, we were witness of how well positioned is FUR within economics and the social sciences, attracting the best talent in the field. That made the task of getting world-class keynote speakers simpler than expected.

This special issue collects a representative sample of what was presented in the conference. As has been customary in the latest editions, we have a mixture of theoretical and empirical work, and both the normative and experimental sides are well represented.

On the theoretical side, we would like to highlight the contribution of Duncan Luce. In Interpersonal Comparisons of Utility for 2 of 3 Types of People, he argues that there is a solution to carrying out interpersonal comparisons of utility when the theory is supplemented with a group operation of joint receipts, and expects that this approach may lay the ground for a new way to make social welfare comparisons.

Other theory articles include that of V.I. Danilov and A. Lambert-Mogiliansky, who in Expected Utility Theory under Non-Classical Uncertainty extend Savage's theory of decision making under uncertainty by replacing the lattice of events by an arbitrary ortho-complemented poset. They claim that any reasonable preference on the set of bets can be represented as expected utility with respect to some belief, that is a probabilistic measure on the orthoposet of events.

This issue also contains some theoretical work at the interface with finance theory. Alexandre Street works on Value-at-Risk models and publishes here his article On the Conditional Value-at-Risk Probability Dependent Utility Function. He links the

M. Baucells $(\varangle) \cdot$ F. H. Heukamp

IESE Business School, 08034 Barcelona, Spain

e-mail: mbaucells@iese.edu

A. Bosch-Domènech

Universitat Pompeu Fabra, 08005 Barcelona, Spain 
Conditional Value-at-Risk measure to Choquet Expected Utility. This allows for a link between a revenue distribution function and local utilities. Street shows the properties of this kind of utility function and a relativistic investment interpretation. Moreover, he works out all the implications for the Allais paradox. The Conditional Value-at-Risk measure also has properties that can be the basis of pricing applications in finance and the basis for simpler multiperiod decision problems.

Multiattribute decision theory, and preferences over consumption, is a topic that, in our opinion, has been under-researched by the field. One possible reason is that there are few normative axioms that restrict preferences beyond transitivity and completeness. However, conditions such as dynamic consistency, and correct predictions of future preferences, do deserve study from both the normative and descriptive side. One article included in this special issue considers multiattribute preferences. If it well known that if we have exponential utility function, then adding a common amount of money to the consequences of a lottery increases the certainty equivalent of such a lottery by that same constant. In In Invariant Multiattribute Utility Functions, Ali Abbas generalizes this type of invariance to multiattribute utility functions, and to general transformations of the outcomes and the certainty equivalents. This finding is useful, as it can be a direct way to elicit the utility functions from clients. If clients agree that their preferences satisfy a particular invariance condition, then we can infer the functional form of their utility function.

Also in the domain of multiattribute decision theory, we have the article A Descriptive Multi-Attribute Utility Model for Everyday Decisions by Jie and David Weiss, together with the late Ward Edwards. They extend the classical multiattribute utility theory by a salience parameter. In this way, the decision maker is a bit more "human." The model is applied to survey data on adolescents' smoking behavior. The implications of the approach go further: Weiss et al. argue that the model gives insight into the dynamics of repeated decisions and personal policy setting.

On the experimental side, we would like to highlight the article A Parametric Analysis of Prospect Theory's Functionals for the General Population by Adam S. Booij, Bernard M.S. van Praag and Gijs van de Kuilen. Adopting the framework of prospect theory, the authors elicit the risk preferences of a large sample of about 2000 Dutch households. They manage to estimate the parameters of curvature of the value function, loss aversion, and curvature-elevation of the weighting function. Each of these parameters influences risk preferences in different ways. Interestingly, they examine which of these parameters depend on population characteristics such as income, gender, education, and age. The question of whether, say, there are gender differences in risk preferences is broken into whether there are differences in the parameter of loss aversion, or whether the curvature of the value function is different. This provides a more accurate view of the effect of population characteristic on risk preferences.

Another domain of decision making that has received limited attention is the area of combined risky and intertemporal choice. Baucells and Heukamp have published a functional representation for simple risky and delayed prospects and publish here an experimental study. In Common Ratio with Delay they show that the common ratio effect can be induced by adding a delay to a choice task. This complements the experimental work by Keren and Roloefsma (1995) and Chapman and Weber (2005) and adds to the view that both attributes of choice, delay and risk, are to a good part inter- 
changeable. This can have an effect on the design of financial products. The authors provide a parameterization of their own model on the basis of the experimental data offering a practical tool.

The introduction of error into a simple model to explain behavioral findings is a parsimonious and fruitful research avenue. One of the pioneers of this line of research is Pavlo R Blavatskyy. Here, we include a sample of his research, coauthored with Ganna Pogrebna. Many behavioral research studies aggregate patterns of behavior, implicitly assuming homogeneous behavior patterns across individuals. In the article Reevaluating Evidence on Myopic Loss Aversion: Aggregate Patterns versus Individual Choices, they examine individual data that violate myopic loss aversion. They propose that a simple Fechner error model satisfactorily explains the evidence from experiments. These same authors in Endowment Effects? Even with Half a Million on the Table! make use of the fascinating data that the TV show "Deal or No Deal" provides to test whether endowment effects are observed when, in the course of the show, contestants are offered to exchange their boxes for other boxes with the same distribution of monetary prizes (that can contain as much as a half million Euros). They find little evidence of endowment effects.

A Fechner error model is also used to answer the question: Are risk preferences stable over time? In Stability of Risk Preferences and the Reflection Effect of Prospect Theory, Baucells and Villasís experimentally document that risk preferences do change when using separate elicitation sessions. They argue that this instability can be attributed to noise, and that around $80 \%$ of subjects exhibit a stable underlying pattern of risk preference, coinciding with the reflection effect of Prospect theory. The other $20 \%$ are risk averse for both gains and losses.

Transitivity is one of the pillars of preference theory, both for decision under certainty and under uncertainty. Amélie Vrijdags in An Experimental Investigation of Transitivity in Set Ranking does precisely this, testing transitivity of preferences when uncertain decisions are described only by all their foreseeable consequences, with no mention of probabilities. She concludes that transitivity seems a plausible condition for the ranking of sets of monetary consequences. Transitivity is also tested in Conflicting Violations of Transitivity and Where They Lead Us by Brett Day and Graham Loomes. They address the conflicting evidence that shows choice cycle consistent with regret theory and choice patterns that contradict it and test whether this is due to different subject pools or experimental environments. They show that the same sample of people operating under the same conditions generate both kinds of cycles. They discuss the implications of such result.

We hope this special issue provides the right palette of what the field does, and indicates where the advances are occurring. What will the future bring? Let us use this opportunity to speculate. The field of decision theory is founded on preference orderings, yielding utility functions as convenient ways to represent these preferences. If the goal is to understand what rationality is, we daresay that a lot has been done. However, if the model is to create approximate models of human behavior for the understanding of actual social systems, then we are still far from reaching the goal.

Rational utility functions cannot directly be interpreted as satisfaction or happiness, or any psychological measure of well-being, and cannot easily be interpersonally compared. However, utility theory appears too detached from what motivates 
humans. Efforts to bring the theory closer to actual preferences and behavior are often orthogonal to the rational model. New evidence confirms that, in many environments, humans are far from exhibiting stable preferences and rational behavior and, due to the heterogeneity of humans, there is doubt that we will ever converge to a satisfactory model of human behavior.

Our guess is that the understanding of human behavior is a multisided bridge, which needs to be started from several ends, each complementing the other. Utility theory is one such end. Another side is closer to where preferences originate, and this involves the study of emotions, and how emotions affect preferences, beliefs, and behavior, and produce "utility" in the sense of Bentham, Jevons, and Edgeworth. This study of emotions can point us to the sources of utility, which certainly may contain many nonconsumption decisions such as the "gratification from overcoming." In this, the new tools of neuroimaging for sure will be helpful.

Another side of the bridge will build on ethical considerations and social preferences and study, with the help of evolutionary biology, how these preferences are formed and impact on decisions.

Learning shall be an important part of the analysis of decision making if we believe, with Lucas, that "people cannot be fooled over and over again," or that individuals use heuristics, shortcuts, simple rules that have proved to provide good results in many situations.

Not to mention that, some of us (economists) are interested in how individual decisions are aggregated in groups and, here, context becomes crucial. Understanding individual decision-making is only part of the answer if we do not know how it aggregates in complex social situations.

The road ahead is as exciting as it used to be 50 years ago, as there are many routes yet to be explored. All we hope is that, as it has done in the past, the field continues to attract the best minds.

\section{References}

Keren, G., \& Roelofsma, P. (1995). Immediacy and certainty in intertemporal choice. Organizational Behavior and Human Decision Processes, 63(3), 287-297.

Weber, B. J., \& Chapman, G. B. (2005). The combined effects of risk and time on choice: Does uncertainty eliminate the immediacy effect? Does delay eliminate the certainty effect. Organizational Behavior and Human Decision Processes, 96(2), 104-118. 